\title{
Instrumentation, control and data acquisition system with multiple configurations for test in nuclear environment
}

\author{
Chiara Monti $^{\mathrm{a}}$, Carlo Neri ${ }^{\mathrm{a}}$, Fabio Pollastrone ${ }^{\mathrm{a}}$ \\ ${ }^{a}$ Associazione EURATOM-ENEA sulla Fusione, ENEA C.R. Frascati, via E. Fermi, 45, 00044 Frascati (Rome) Italy
}

The In-Vessel Viewing System is a 3D laser scanning system which will be used to inspect the blanket first wall in ITER. To make the IVVS probe design compatible with the harsh environmental conditions present in ITER, a test campaign was performed in 2012-2013 to verify the adequacy of the main components of the IVVS probe. The IVVS components inspected were an optical encoder, passive components and two customized ultrasonic piezoceramic motors that were instrumented with various sensors. A general architecture of the Data Acquisition and Control System (DACS) was defined and then specialized for each test. To be suitable for this test campaign, the DACS had to host various I/O modules and to properly interface the driver of the customized piezo motors, in order to permit the full control of the test and the acquisition of experimental data. This paper presents the instrumentation solutions designed and implemented for different facilities constraints and the related DACS developed in four specialized versions for the described test campaign.

Keywords: ITER; IVVS; data acquisition; radiation resistant; piezoceramic.

\section{Introduction}

The In-Vessel Viewing System (IVVS) is a 3D laser scanning system which will be used to inspect the first wall in ITER. The IVVS will be stored inside a port plug, and will be deployed inside the vacuum vessel on demand, mainly for diagnostic and maintenance purposes. The IVVS will permit to make erosion evaluation of the in-vessel surfaces providing high resolution images [1]. A first prototype of the IVVS probe was designed and constructed by ENEA staff during 2001 [2]-[6]. Since that first prototype, several activities have been carried out to make the IVVS probe compatible with the harsh environmental conditions in ITER and with the design constraints [7]. During 2012 and 2013, in the framework of a Fusion for Energy (F4E) Grant agreement, testing activities were performed to verify the compatibility of the IVVS probe main components with the presence of neutrons, gamma radiations, high temperature, vacuum and high magnetic fields. Probe components such as optics, fibers, cables, sensors, connectors, including two piezoelectric motor were tested (see section 2). The experimental campaign involved people having different expertise in laser, optics, mechanics, superconductivity, electronics, analysis of material in rad-hard environment, vacuum and outgassing issue. In the following, the test instrumentation solution, the data acquisition and control issues involved in this experimental campaign will be presented.

\section{Motor tested}

The motors which drive the scanning head are the only active components in the IVVS probe. The IVVS probe has to withstand all the ITER in vessel environmental conditions, therefore the motors are the most critical components. Due to the presence of high magnetic field, ultrasonic piezo motor technology was chosen as a valid candidate to cope with the harsh environment of the ITER vessel. To validate the piezo motor technology a test campaign was performed on two custom rotary stages. Each rotary stage is driven by two PILine ultrasonic piezomotors and differs from the other only for the dimensions; for the sake of simplicity we will refer to the rotary stage as the piezo motor. M$660 \mathrm{~K} 044$ has the typical dimensions of the motor to be used in the real application (square section is $115 \times 115$ $\mathrm{mm})$, while the smaller one (M-660K045) is characterized by a circular section of $50 \mathrm{~mm}$ of diameter (Fig. 1).

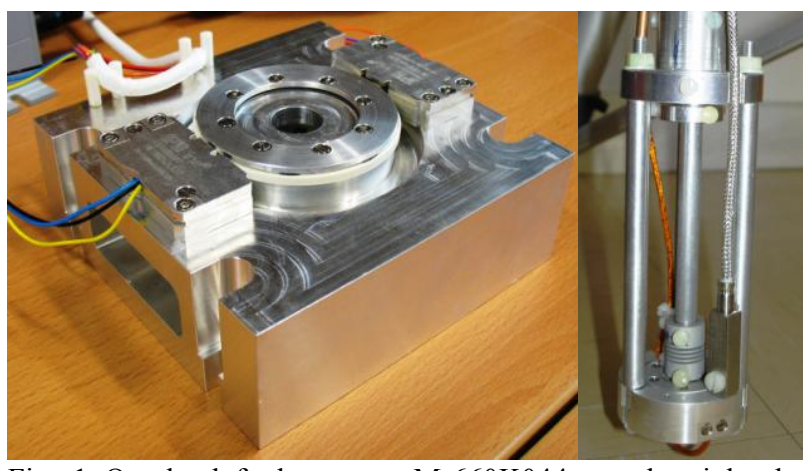

Fig. 1 On the left the motor M-660K044, on the right the smaller motor M-660K045 in the mechanical assembly used for the magnetic test compatibility

\section{Tests overview}

The test campaign had to demonstrate that the piezoceramic motor technology works properly in ITER environmental conditions:

- High vacuum (HV) conditions: $5 \times 10^{-4} \mathrm{~Pa}$.

- Operating temperature: $\leq 120^{\circ} \mathrm{C}$.

- Baking temperature: up to $200^{\circ} \mathrm{C}$.

- Magnetic field: up to $8 \mathrm{~T}$ in deployed position

- Gamma dose rate: up to $2 \mathrm{kGy} / \mathrm{h}$ (probe only, during in-vessel deployment).

- Gamma radiation integrated lifetime dose: up to 4 MGy (probe only).

- Cumulative neutron fluence: up to $2.3 \times 10^{17} \mathrm{n} \mathrm{cm}^{-2}$ (cumulated after $20 \mathrm{yrs}$ of operation).

- Neutron flux spectrum: from $10^{-7} \mathrm{MeV}$ to $2 \mathrm{MeV}$.

The tests were performed in different facilities of ENEA laboratories. Four tests were scheduled:

author's email: chiara.monti@enea.it 
- compatibility of the piezo motor with gamma irradiation, performed in the CALLIOPE facility in ENEA Casaccia;

- compatibility of the piezo motor with multi-tesla magnetic field, performed in a superconducting facility in ENEA Frascati;

- compatibility of the piezo motor with neutron irradiation, performed in the TAPIRO research reactor in ENEA Casaccia;

- compatibility of the piezo motor with high vacuum conditions and high temperature, performed in a dedicated vacuum facility in ENEA Frascati.

Each facility is characterized by different constraints and environmental conditions, that have influenced how the test sample was instrumented. Both the superconducting solenoid magnet and the TAPIRO Research Reactor can host a sample having a maximum diameter of $55 \mathrm{~mm}$, so the small motor was used, while the CALLIOPE and the vacuum facility allowed testing the large motor. The use of the two motors was also crucial to be able to complete the activities within the short time required. In this way it has been possible to prepare and carry out two tests in parallel, thus optimizing the time.

\section{Instrumentation of the motors and Data Acquisition and Control issue}

Since the motors had to be tested in ITER environmental conditions (see section 1), then also the sensors used in the test had to withstand the same environment, in particular for doses of radiation and high temperatures. Specific sensors previously developed within the IVVS project to withstand ITER conditions and commercial sensors were used. The commercial sensors were chosen resistant to high temperature, having these more possibility to resist high dose of irradiation. Depending on the facility constraints and the specific test procedure, different sensors have been used: encoders, a rheostat, switches and a torque transducer. Furthermore, each test was instrumented in order to measure the environmental parameters: temperature, pressure, magnetic field depending on the kind of test.

All the electronics that allow collecting the signals from the sensors, were placed in a shielded area having standard room conditions. Thanks to this, it was possible to choose a COTS DAQ and the commercial driver of the piezo motor. A general layout common to the two irradiation test was identified and consists in two subassembly and the connection cables (optical and electrical cables). In Fig. 2-A the specific layout of the gamma test is shown.

The choice of the commercial hardware to perform the data acquisition was led by the following wants.

- A general purpose DAQ platform, in order to acquire analog or digital signals from several different sensors.

- A compact size and a rugged design, in order to easily move the system in the different test facilities.

- A reduced software development time and easy DAQ hardware structure configuration, because the time schedule was very tight.
Furthermore, a key requirement for the DACS system was that it had to allow online analysis, presentation and storing of the data during the acquisition, creating a database to permit offline analysis. The DACS system would have to permit remote control and monitoring of the test.

Having planned two parallel tests in facilities located in different sites, four DAQ systems were foreseen: two online DAQ systems, one for development purpose and one for backup.

Having these requirements, the choice of the data acquisition hardware fell on a compactDAQ (cDAQ) from National Instruments (NI); cDAQ is a chassis that supports up to 8 slots where each slot can hold an input/output (I/O) module. The flexibility in $\mathrm{I} / \mathrm{O}$ modules made it ideal for this test campaign. The typical solution for the data logging issue is to connect the system to a personal computer where the data acquired were logged. The cDAQ offers communications to PC through USB, ethernet or $\mathrm{Wi}-\mathrm{Fi}$; in all tests the USB communication was used, but also an Ethernet-cDAQ was purchased when all facility and motor controller constraints were not available.

In order to achieve the specific configuration required for each test, the following I/O modules were purchased:

- NI 9215 that includes four simultaneously sampled analog input channels at $100 \mathrm{kS} / \mathrm{s}$ per channel, \pm 10 $\mathrm{V}$ measurement range and 16-bit resolution.

- $\quad$ NI 9217 that is a resistance temperature detector (RTD) analog input module; it features 4 channels at $400 \mathrm{~S} / \mathrm{s}(100 \mathrm{~S} / \mathrm{s}$ per channel) and 24 bits of resolution.

- $\quad$ NI 9211 thermocouple input module that includes 4 channels at $14 \mathrm{~S} / \mathrm{s}, \pm 80 \mathrm{mV}$ measurement range and 24-bit resolution.

- $\quad$ NI 9401 that is an 8-channel, 100 ns bidirectional digital IO module. Each channel is compatible with $5 \mathrm{~V} / \mathrm{TTL}$ signals and features 1,000 Vrms transient isolation between the $\mathrm{I} / \mathrm{O}$ channels and the backplane. In this test campaign the NI 9401 was used always in input configuration.

To achieve the acquisition, storage, processing and presentation of the data through a friendly user interface, the LabVIEW software was used. LabVIEW is a graphical programming environment supplied by NI that is totally integrated with the hardware chosen. LabVIEW has enabled rapid development of the system and high flexibility in the improvement and adjustment of the system during the test. By now it is considered a standard in the field of measurements and control system; the producer of the piezo motors supplied also a LabVIEW driver library to be used to control the C-867 Piezo-motor Controller.

\section{Compatibility with Gamma irradiation}

The target of the test was to evaluate the reliability and lifetime expectation under gamma radiation up to 4 MGy of the large motor M-660K044. The test was performed in the CALLIOPE facility [8].

As explained in section 4 and outlined in Fig. 2-A, the motor was instrumented in rad hard way by placing 
all the electronics in the shielded area. The irradiated components, shown in Fig. 2-B-C, were an encoder optical system, a rheostat used like an angular position transducer and as a resistant element that gives a resisting torque of $0.1 \mathrm{Nm}$, a couple of switches to give an additional resisting torque of $0.09 \mathrm{Nm}$ and a temperature sensor RTD. The encoder optical system was composed by a gear where teeth were optically read by a couple of optical fibers silica/silica 600 micrometer core. Having done a risk analysis, the test has been devised taking into account the likelihood that the sensors in use could be damaged or degraded during irradiation. The optical system was very robust; it was however duplicated by the rheostat and the switches, which besides providing a resisting torque, had the purpose of providing information on the speed of the motor. All the electronics interfaces were placed in the shielded area, $18 \mathrm{~m}$ far from the instrumented motor under irradiation; the electronics were the encoder electronics, the rheostat supply, the motor controller PI C-867 and the cDAQ system equipped with three I/O modules shown in Fig. 2-A. The encoder electronics is not commercial and was developed in ENEA; it consists of a Receiving System and a Transmission System. The Transmission System includes a laser diode controller board with power regulator. The Receiving System converts the light returning from the encoder through the optical fibre reception signal in electrical signal. The input stage of the Receiving System is based on an amplified photodetector, necessary to amplify the very low level optical signal. Radiation resistance optical fibers and electrical cables Kapton ${ }^{\circledR}$ insulated were used to connect the instrumented motor to the electronics located in the shielded area. The control room of the facility is in the same building at a distance of $30 \mathrm{~m}$ from where the electronics were located; the motor was controlled and monitored both from the local control room and remotely from ENEA Frascati laboratories through the LabVIEW dedicated control and acquisition system.

The test procedure consisted in running the motor during the gamma irradiation, twice a day for about 1 hour. An algorithm was implemented to run the motor clockwise and counterclockwise, driving the rheostat and pressing two switches; this procedure permitted to verify that the motor was able to overcome an effective torque of $0.19 \mathrm{Nm}$. The test lasted 66 working days and the motor ran 98 hours and turned more than 500 thousand times. The rheostat, which turned together with the motor, worn out due to the very large number of turns performed (Fig. 2-B-C shows the rheostat before and after irradiation). The success of the experimental test campaign was assured thanks to the preventive action of doubling the sensors. The total absorbed dose obtained was of $4882 \mathrm{kGy}$ exceeding the requested dose of 4 MGy. The results collected, detailed in [9], indicates that gamma irradiation did not cause damages to the piezoceramic motor which compromise the proper operation of the motor itself.

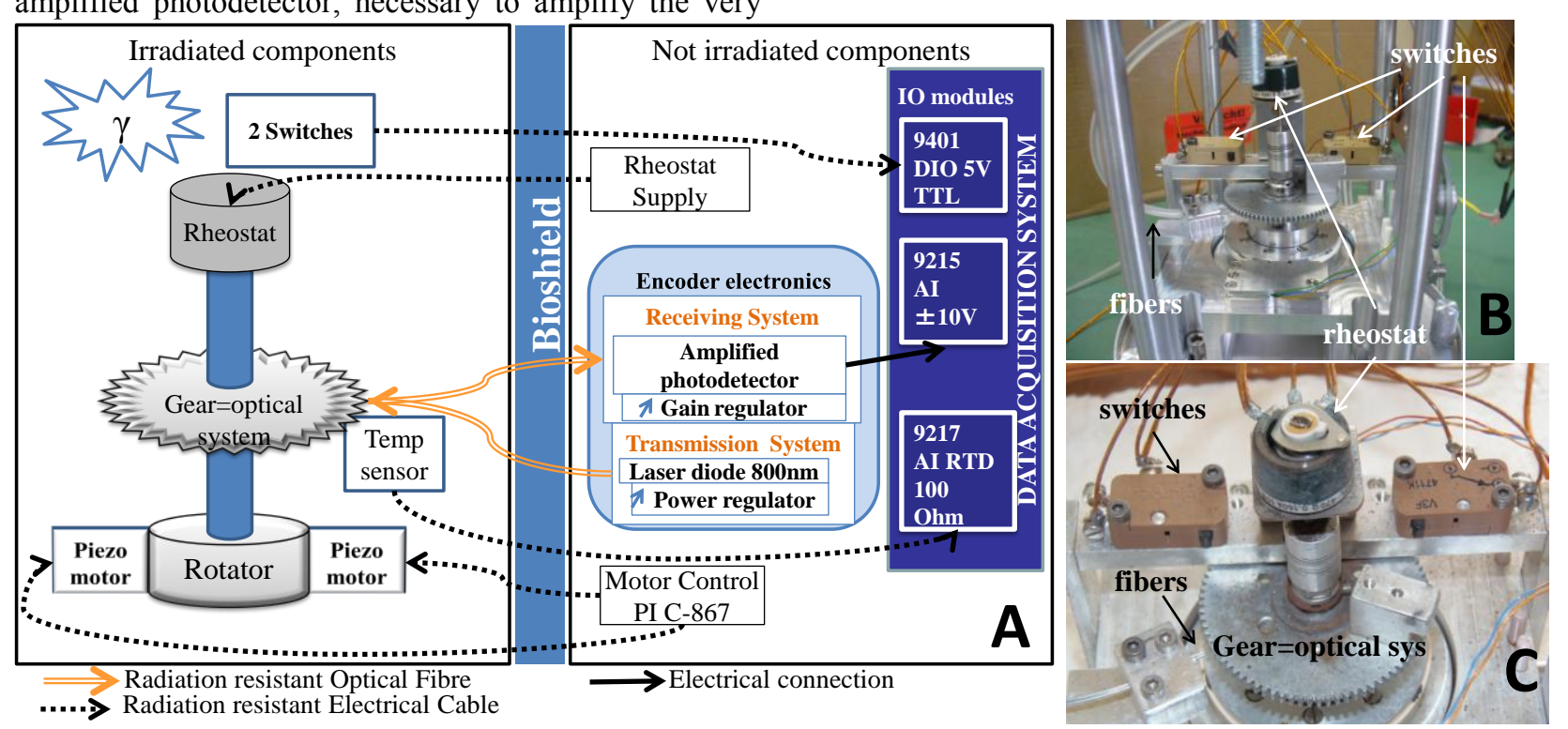

Fig. 2 A: Test layout design for the gamma irradiation B: Close-up of the motor instrumented: the gear read by a couple of fibers, the rheostat and the two switches are shown C: Close-up after irradiation

\section{Compatibility with neutron irradiation}

The experimental facility used is the fast source reactor TAPIRO. The piezomotor irradiated was the one having $50 \mathrm{~mm}$ of diameter to fit the radial channel available, where the motor was subjected to an intense neutron flux which permitted to reach the required cumulative neutron fluence of $2.3 \times 10^{17} \mathrm{n} \mathrm{cm}^{-2}$ in a reasonable time. The irradiation strategy is detailed in [10]. The motor was instrumented and assembled to be remotely monitored and controlled in rad-hard way as in gamma irradiation test described in previous section. Due to the reduced size of the motor it was not possible to duplicate sensors and the motor movement was measured only by the encoder optical system; nevertheless, to mitigate the risk of losing the encoder information, a second pair of fibers was added to the setup. To monitor the motor temperature a previously calibrated thermocouple type $\mathrm{K}$ was used. This thermocouple was calibrated against a certified 
thermocouple by using a National Instruments calibrated 9211 module.

A proper characterization of the piezo-motor was carried out during each shutdown period of TAPIRO.

Two kind of characterisation were implemented and performed. One aimed to find out the characteristic motor speed versus motor driving power, exploring the operative range of motor driving power values. For each selected motor driving power value, the motor was left running for at least 20 seconds to exclude transient situations. The motor speed, the motor driving frequency, the input driving power and the motor temperature were acquired, monitored and recorded during this period; the motor speed was obtained in turn $/ \mathrm{s}$ through a real time processing of the raw data obtained from the encoder. The second characterization was aimed to find out the characteristic motor speed versus resonance frequency, which is an indirect method to find out the resonance curve of the piezoceramic. This method allows also the evaluation of the motor effectiveness. During the characterization the excitation frequency of the driver was recursively incremented with a step of typically $0.025 \mathrm{kHz}$ inside the operative range, for a given motor driving power; during the initial characterization the operative range was from $211 \mathrm{kHz}$ to $216 \mathrm{kHz}$, but it changed during the irradiation. Speed and frequency were acquired during this characterization allowing the evaluation of the optimal resonance frequency. This test permitted to evaluate a possible dependence of the resonance frequency of the ceramic material from the neutron fluence and consequently to use the optimal excitation frequency during the characterization motor speed versus motor driving power, then optimizing the motor performance.

The motor was activated remotely through the LabVIEW user interface, at the beginning of every procedure, and the monitoring was done online during the motor running.

The irradiation campaign has shown that the effect of neutron irradiation up to $2.77 \times 10^{17} \mathrm{n} \mathrm{cm}^{-2}$ produced a shift of the optimal resonance frequency (about $1.25 \%$ ) and a decrease of the maximum motor speed of about $42 \%$. Graphs of the two kind of characterizations together with the detailed description of the neutron test campaign are reported in [10].

\section{Compatibility with multi Tesla magnetic fields}

The test was performed in a superconducting solenoid magnet in ENEA Frascati laboratories, able to reach a magnetic field of $14 \mathrm{~T}$. The magnet is placed into a cryostat and the available space for the test sample is a cylinder of $\varnothing 55 \mathrm{~mm}$, so the piezo motor used was M$660 \mathrm{~K} 045$.

The target of the test was to verify if the motor behavior was affected by ITER relevant magnetic field in steady state condition (8 T) or in slowly varying field. During the test, the speed and temperature of the motor and the magnetic field strength (in the form of magnet supply current) were measured, monitored and stored. To that purpose the motor was instrumented with a Kübler incremental encoder type 3700/3720 that was used as an angular position transducer of the motor, an Electrotherm RTD temperature sensor operating up to $400^{\circ} \mathrm{C}$ used to monitor the motor temperature, an auxiliary motor and a Burster precision torque sensor model 8661 both used to measure the torque applied to the motor due to the shaft. The active instrumentation (encoder, torque sensor and auxiliary motor) was placed outside the magnetic field region.

The cDAQ-9178 hardware was personalized for this test with four I/O modules: NI 9401 to acquire encoder signal, NI 9217 to acquire temperature from the RTD sensor, two analog input modules NI 9215 to acquire torque information and the magnetic field obtained from the magnet supply current. A LabVIEW application was developed and run on a PC where the operator can control the motor, then acquire and monitor all relevant data. The application saved all the acquired data in three kind of $\log$ files, depending on different acquisition rate.

The customized piezo motor was tested up to $10 \mathrm{~T}$, and demonstrated to work properly under magnetic field at a value of $\mathrm{B}$ equal or higher than $8 \mathrm{~T}$ (ITER) for a significant time duration, without any evident damage or decrease of performances. Detailed description of the test and its results are reported in [11].

\section{Compatibility with high vacuum and high temperature}

The test was designed to evaluate the behavior of the motor M-660K044 up to a working temperature of 120 ${ }^{\circ} \mathrm{C}$, a baking temperature of $200{ }^{\circ} \mathrm{C}$ and under a vacuum of $\leq 10^{-2} \mathrm{~Pa}$ that is considered adequate to test the motor operation from the mechanical point of view. A dedicated vacuum facility to test the motor was arranged. This custom motor was previously tested under gamma radiation (see section 5). As in previous tests, the motor was instrumented with an encoder optical system composed by a gear, mounted on the motor, where teeth are optically read by a couple of optical fibers. To measure the pressure inside the vacuum chamber was used a full range pressure gauge Pfeiffer PKR, calibrated against a certified pressure gauge. Then, two thermocouples type $\mathrm{K}$ were used to monitor the temperature of the motor and of the vacuum chamber.

The cDAQ system for this test consisted of three analog input modules: NI 9211 to acquire temperatures from thermocouples, two modules NI 9215 to acquire the motor speed from the encoder signal and the vacuum pressure from the full range gauge. The cDAQ was connected to a personal computer in which the data was stored. Both the data acquisition system and the personal computer were located in the Ex-Vacuum area. The data acquisition policy was to acquire and store all measurements data during each motor run. The data acquired were monitored online through the LabVIEW graphical user interface (GUI) developed to present temperatures, pressure in $\mathrm{Pa}$ of the vacuum chamber and the speed of the piezo motor. Motor speed was obtained in turn/s through a real time processing of raw data obtained from the encoder; raw data was stored in an auxiliary file due to the large amount of data. The pressure was acquired and logged as voltage output from the pressure gauge through the dual gauge controller; the 
coefficients to convert Volt to Pascal was logged, then both the raw pressure data and the converted one were available. Temperatures were logged and reported as they were (no processing needed). Storing both raw data and some processed data was a strategy used in all the four tests in different environment allowing successive additional post processing and offline analysis.

The GUI permitted the complete control of the test parameters, and the storage of the operator input parameters in the database in order to allow an effective post analysis of the experimental data, then making possible the causal relation between input and output data.

Once installed inside the vacuum facility, the motor was preliminary characterized at air pressure and ambient temperature. Then vacuum was made in the chamber down to a pressure of about $10^{-4} \mathrm{~Pa}$ and the temperature was raised up to $120^{\circ} \mathrm{C}$ in steps of $10{ }^{\circ} \mathrm{C}$; the piezoelectric motor was able to run in each temperature step. A dependency of the motor behavior and of the resonance frequency from the temperature was verified, consequently a tuning of the resonance frequency is necessary when using the motor at high temperature.

\section{Conclusion}

The design, instrumentation solution and setup of this test campaign permitted to validate the piezo motor technology in an effective way. The test campaign has exploited some of the technical background developed during IVVS project, such as the use of rad-hard optical fibers based sensors. Then further to the piezo motor technology, the test campaign has also validated instrumentation solutions which can be effectively used in final IVVS implementation or other ITER diagnostics or applications.

\section{Acknowledgments}

The work leading to this publication has been funded partially by Fusion from Energy under the Grant F4EGRT-282. This publication reflects the views only of the author, and Fusion for Energy cannot be held responsible for any use which may be made of the information contained therein

\section{References}

[1] F. Pollastrone et al., Erosion evaluation capability of the IVVS for ITER applications, Fusion Eng. Des. 89(2014) http://dx.doi.org/10.1016/j.fusengdes.2014.03.058.

[2] A. Coletti et al., AM laser system (IVVS) for the ITER in vessel viewing and ranging, Fusion Eng. Des. 69(2003) http://dx.doi.org/10.1016/S0920-3796(03)00312-0.

[3] C. Neri et al., Experimental result of the laser in vessel viewing and ranging system (IVVS) for ITER, Fusion Eng. Des. 75-79 (2005), Pages 613-618, ISSN 0920-3796, http://dx.doi.org/10.1016/j.fusengdes.2005.06.185.

[4] C. Neri et al., The laser in vessel viewing system (IVVS) for ITER: test results on first wall and divertor samples and new developments, Fusion Eng. Des. 82(2007) http://dx.doi.org/10.1016/j.fusengdes.2006.12.006.

[5] C. Neri et al., The upgraded laser in vessel viewing system (IVVS) for ITER, Fusion Eng. Des. 84 (2009) http://dx.doi.org/10.1016/j.fusengdes.2009.01.096.

[6] C. Neri et al., Iter in vessel viewing system design and assessment activities, Fusion Eng. Des. 86 (2011) http://dx.doi.org/10.1016/j.fusengdes.2011.02.047.

[7] C. Neri et al., Compendium of the experimental and design activities toward the manufacture of the In Vessel Viewing System for ITER, this conference

[8] S. Baccaro, A. Cecilia, A. Pasquali, Gamma irradiation facility at ENEA-Casaccia Centre (Rome), ENEA RT/2005/28/FIS, ISSN/0393-3016 (2005).

[9] P. Rossi et al., IVVS actuating system compatibility test to ITER gamma radiation conditions, Fusion Eng. Des. 88 (2013) http://dx.doi.org/10.1016/j.fusengdes.2013.03.030.

[10] M. Pillon et al., Study of the response of a piezoceramic motor irradiated in a fast reactor up to a neutron fluence of $2.77 \mathrm{E}+17 \mathrm{n} / \mathrm{cm}^{2}$, this conference

[11] C. Monti et al., Test of piezo-ceramic motor technology in ITER relevant high magnetic fields, Fusion Eng. Des. 89 (2014) http://dx.doi.org/10.1016/j.fusengdes.2014.04.023 
